- became clear that setting up the centre would mean dismantling and dividing up the pieces of the National Center for Research Resources, a \$1.3-billion $\mathrm{NIH}$ institute devoted to infrastructure and training. In Congress, Rehberg and others have questioned whether the agency is pushing the government into an area drug development - that they say should be the preserve of industry. Collins has said that NCATS will "complement — not compete with" the private sector.

On 15 June, after Collins announced a search for a director of the new centre, Rehberg wrote to Kathleen Sebelius, who heads the Department of Health and Human Services, the NIH's parent agency, pronouncing himself "very troubled" by the search. Without an official budget amendment outlining the financing of the centre and explaining other details of the NIH's plans that would "allow us to review the proposal fully", Rehberg wrote, "we cannot responsibly take any action on this matter".

Under the Freedom of Information Act, Nature has obtained Sebelius's 6 July response to Rehberg's letter, which ran, with attachments, to 79 pages of minute detail about the structure, finances and goals of the proposed centre (see go.nature. com/4wzijl). In her covering letter, Sebelius told Rehberg that the NIH has confined itself to "preliminary planning" with the aim of an efficient launch in 2012, and adds that the agency will not hire a director "until NCATS is established". Rehberg has not responded to multiple requests for comment over the 12 weeks since he wrote to Sebelius.

But Congress's broader budget troubles seem as likely to delay NCATS as the skirmish with Rehberg. David Moore, a veteran congressional observer and senior director for governmental relations at the Association of American Medical Colleges in Washington DC, says that the increasingly charged atmosphere around spending issues on Capitol Hill makes it impossible to predict the chances of Congress passing a bill - whether a stop gap measure or a full 2012 law - in time for NCATS to launch on 1 October. "We think it's going to happen. We just don't know how or when," he says.

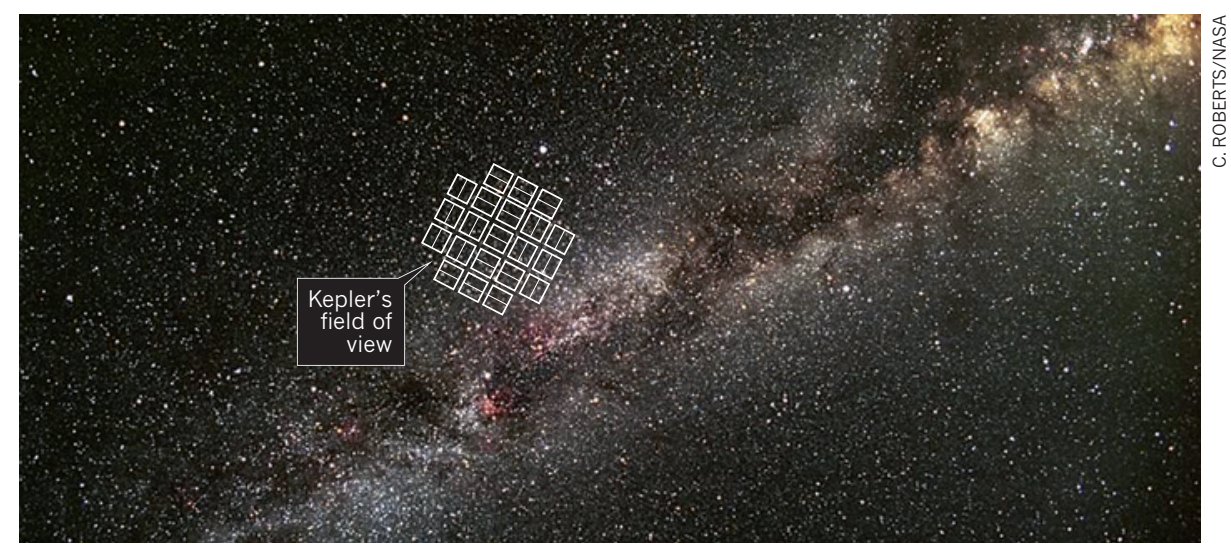

The Kepler probe is searching part of the Milky Way for signs of Earth-like planets.

ASTRONOMY

\title{
Jumpy stars slow hunt for other Earths
}

\section{NASA mission looks for extra time to battle stellar noise.}

\section{BY RON COWEN}

$\mathrm{T}$ The Kepler spacecraft has hit an unexpected obstacle as it patiently watches the heavens for exoplanets: too many rowdy young stars. The orbiting probe detects small dips in the brightness of a star that occur when a planet crosses its face. But an analysis of some 2,500 of the tens of thousands of Sunlike stars detected in Kepler's field of view has found that the stars themselves flicker more than predicted, with the largest number varying twice as much as the Sun. That makes it harder to detect Earth-sized bodies.

As a result, the analysis suggests that Kepler will need more than double its planned mission life of three-and-a-half years to achieve its main goal of determining how common Earthlike planets are in the Milky Way.

Kepler is currently slated to run until November 2012 and, given NASA's budget pressures, including the ever-rising cost of the James Webb Space Telescope (JWST), an extension is by no means certain. "I'm concerned that programmes like Kepler might be squeezed out in order to have enough funding for the JWST," says Kepler's chief data analyst, Jon Jenkins at the SETI Institute in Mountain View, California. "We need an eight-year mission to reach our goal of understanding whether there are other worlds out there like Earth." NASA declined to comment on Kepler's chances of securing an extension; a decision is expected next spring.

In many ways, the mission has been a huge success. Earlier this year, Kepler scientists announced that the craft had discovered 1,235 candidate exoplanets, 68 of which are Earthsized. Fifty-four of the total number of planets detected, all of them larger than Earth, orbit within the habitable zone of their parent stars, a region temperate enough for water to remain liquid on the surface of a rocky body. More exoplanet discoveries are expected to be announced next week in Moran, Wyoming, at a conference on extreme solar systems, and a new batch of Kepler data, based on 674 million observations recorded from September to December 2009, is scheduled for public release on 23 September.

In an article posted online in July and

\section{TOP STORY}

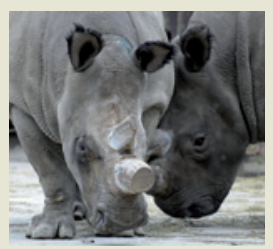

Could stem cells rescue an endangered species? go.nature.com/ v69zxu

\section{MORE NEWS}

- Electrified bacterial filaments zap uranium go.nature.com/q30dag - Oil-spill research funds for Deepwater Horizon begin to flow go.nature.com/vnapdj

- Chinese science ministry increases funding go.nature.com/h1kslk

\section{ON THE BLOG}

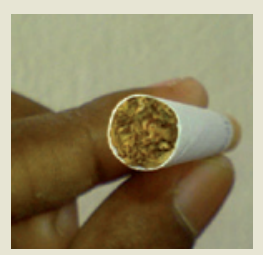

Tobacco company seeks access to secret data on smoking habits go.nature. com/5cwhsx 
¿ currently in press in The Astrophysical Journal, Kepler scientist Ron Gilliland of the Space Telescope Science Institute in Baltimore, Maryland, and his collaborators describe Kepler's noise dilemma (R. L. Gilliland et al. preprint at http://arxiv.org/abs/1107.5207; 2011). The signal of an Earth analogue is a drop in a star's luminosity of about 85 parts per million, lasting several hours, whenever such a planet passes in front of its parent star.

Before Kepler's launch in early 2009, the team had assumed that the Sun-like stars viewed by the craft would be about as quiet as the Sun, with luminosity fluctuating in a range near 10 parts per million (a $0.001 \%$ variation) over the key timescale of about 6.5 hours. But Gilliland and his colleagues found that the noise in the Kepler data is much larger (see 'Too much twinkle'), with most of it intrinsic to the stars themselves, rather than caused by instrument problems or other errors associated with recording low light levels.

Gilliland believes that the stellar equivalent of sunspots are the most likely culprits. Sunspots form when magnetic activity creates areas

$\rightarrow$ NATURE.COM on the solar surface that

For more on Kepler's hunt for other Earths, see: go.nature.com/qoydhc

\section{TOO MUCH TWINKLE}

In a sample of 2,500 Sun-like stars monitored by the Kepler probe, most vary in brightness more than the Sun does, which makes planets harder to see.

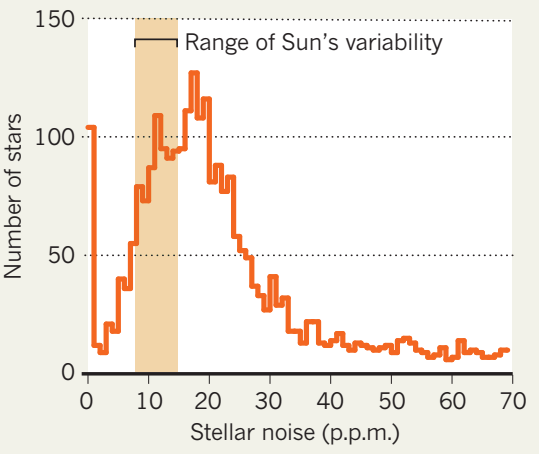

activity is higher among the Sun-like stars in the Kepler field than in the Sun itself, probably because they are younger. Younger stars spin faster, driving a more vigorous magnetic field.

In fact, Gilliland suggests that about half the stars in the Kepler field are younger than the Sun, even though theoretical predictions had indicated that about two-thirds would be older. If this youthful bias is true of the entire Milky Way, "that would indeed be a surprise", with far-reaching implications for how stars are born and die, Jenkins says.

But whatever the source of the stellar noise,
Gilliland says that Kepler will have to detect twice as many transits of Earth-like analogues than planned - an average of six passages per planet rather than three, at intervals of roughly a year - to be sure that a dip in starlight is a true signal of an Earth-sized body. This means that the probe will need a full eight years to attain its original goal of finding about $75 \%$ of Earth analogues among its target stars.

"We need an extended mission because the detection of Earth-sized planets hangs in the balance," says Geoff Marcy an astronomer at the University of California, Berkeley, and a member of the Kepler team.

Focusing on planet candidates that have a diameter no smaller than 1.2 times that of Earth could speed up the mission, says Gilliland, because they cast a deeper shadow and so are easier to pick out from the stellar noise. But a planet that is 20\% larger than Earth may not be a rocky body like our home planet, some models suggest. It could, for instance, be a 'waterworld', with a deep ocean or a shell of ice.

If the mission is restricted to such larger planets, it may not answer the question that Kepler was built to study, says Gilliland. Finding out whether other Earths exist "is a question humans have been asking for thousands of years", says Jenkins. "It would be a real shame" if NASA ends the mission "just as Kepler is on the threshold of answering it". 